\title{
Síntesis de un polímero biodegradable, como alternativa a los polímeros sintéticos
}

\author{
Johana Troya, Roman Rodríguez, Daniela Calvache \\ Departamento de Energía y Mecánica, Ingeniería Petroquímica, Universidad de las Fuerzas Armadas ESPE-L. \\ johastr@hotmail.com,rnrodriguez@espe.edu.ec,danita_tefa@hotmail.es
}

\begin{abstract}
Resumen-El presente estudio aporta información sobra la síntesis de un polímero biodegradable como una alternativa a los polímeros sintéticos, los cuales tardan en degradarse varios años y son de origen petroquímico. El ácido poliláctico (PLA), se obtuvo a partir de ácido láctico, mediante los métodos: Policondensación con catalizador y Policondensación sin catalizador, de lo cual de comprueba que mediante la formulación de ambos métodos es factible la síntesis de PLA. Para el método de policondensación con catalizador las variables que fueron estudiadas y las condiciones del método son: concentración de catalizador $(6 \%$ en peso de $\mathrm{Zn})$, temperatura $\left(180{ }^{\circ} \mathrm{C}\right)$, tiempo de reacción (8 horas); mientras que las variables y las mejores condiciones obtenidas para el método de policondensación sin catalizador son: temperatura $\left(180{ }^{\circ} \mathrm{C}\right)$, tiempo de reacción (20 horas). De los experimentos realizados se calcula el rendimiento obtenido en la síntesis de PLA con catalizador, el cual es de $67,53 \%$, mientras que el rendimiento para el método libre de catalizador es $68,83 \%$. El PLA que se sintetizó por cada uno de los métodos es analizado por medio de tres métodos de análisis: espectroscopia infrarroja con transformada de Fourier (FTIR), Termogravimetría (TGA) y Calorimétria Diferencial de Barrido (DSC).
\end{abstract}

Palabras Claves-Ácido láctico, ácido poliláctico, biodegradación, biopolímero.

Abstract-The present study provides information on the synthesis of a biodegradable polymer as an alternative to synthetic polymers, which take several years to degrade and are of petrochemical origin. Polylactic acid (PLA), was obtained from lactic acid, by the methods: Polycondensation with catalyst and Polycondensation without catalyst, from which it verifies that by the formulation of both methods the synthesis of PLA is feasible. For the method of polycondensation with catalyst the variables that were studied and the conditions of the method are: catalyst concentration $(6 \%$ by weight of $\mathrm{Zn})$, temperature $\left(180^{\circ} \mathrm{C}\right)$, reaction time $(8$ hours); while the variables and the best conditions obtained for the polycondensation method without catalyst are: temperature $\left(180{ }^{\circ} \mathrm{C}\right)$, reaction time (20 hours). From the experiments carried out, the yield obtained in the synthesis of PLA with catalyst is calculated, which is $67.53 \%$, while the yield for the free catalyst method is $68.83 \%$. The PLA that was synthesized by each of the methods is analyzed by means of three analysis methods: infrared spectroscopy with Fourier transform (FTIR), Thermogravimetry (TGA) and Differential Scanning Calorimetry (DSC).

Keywords-Lactic acid, polylactic acid, biodegradation, biopolymer.

\section{INTRODUCCIÓN}

La riqueza de un país, en primera instancia, depende de la capacidad de este para producir y comercializar bienes y servicios, la transformación de la matriz productiva se ve direccionada al respeto de los derechos tanto de la naturaleza como de la justicia intergeneracional [1].
En la actualidad, se necesita la implementación de industrias nuevas o mejoradas, que tengan como objeto reducir el impacto ambiental al momento de la elaboración de productos finales y que a su vez estas industrias se encuentren diversificadas, con la finalidad de satisfacer las necesidades de los seres vivos y alcanzar la reducción del impacto ambiental [2].

El daño del uso indiscriminado de los recursos derivados del petróleo se ha podido evidenciar de mejor manera en los últimos años, no solo en el aspecto de que este es un recurso natural no renovable, sino también en el impacto ambiental que ha generado tanto en aire, agua y suelo, razón por la cual se busca promover el avance tecnológico tanto de biorefinerías como de la química verde [3], [4].

Los polímeros naturales generan mejor contribución al término de biodegradabilidad; mientras que los polímeros sintéticos que aportan a esta propiedad se han incrementado notoriamente, analizando propiedades que permiten mejorar la degradación al momento de convertirse en residuos [5].

El PLA es una macromolécula, la cual se encuentra formada por moléculas de ácido láctico, presenta variadas características que lo hacen interesante, como poseer propiedades semejantes a las del tereftalato de polietileno (PET) y la ventaja que este es un material que es biodegradable, el cual llega a desintegrarse con myca facilidad en agua y óxido de carbono, presenta además una llamativa aplicación dentro de su utilización en las impresiones 3D [6].

La síntesis de PLA es una opción al momento de la elaboración de polímeros que son biodegradables. Es empleado para la elaboración de empaques de productos secos como almendras, pistachos, maní, además, es de mucha utilidad en la elaboración de materiales de sutura reabsorbible usado en diferentes tipos de cirugías como oftalmológicas u ortopédicas, se aplica además para microencapsular y nanoencapsular medicamentos de liberación lenta como la insulina u antiinflamatorios, entre otras aplicaciones [7], [8].

Se han observado varias mejoras dentro de la industria petroquímica del plástico, la cual posee alrededor de 100 años de existencia, mientras que la síntesis de PLA posee alrededor de 10 años de fabricación y aún se encuentra en progreso de mejora, lo cual hace del PLA un producto de interés [9].

Este trabajo de investigación propone el estudio de la síntesis de un material biodegradable, como sustituto de los polímetros tradicionales sintéticos, con el objeto de brindar un aporte a posibles soluciones del impacto ambiental, que es causado por el uso de los derivados del petróleo, y así impulsar futuras investigaciones las cuales centren la atención al progreso de materiales amigables con el medio 
ambiente, aptos para aplicaciones petroquímicas, permitiendo el aprovechamiento de los recursos naturales renovables, fomentando el bienestar actual y futuro de los recursos e impulsando la química verde.

\section{MÉTODO}

\section{A. Materiales}

Se empleó Ácido Láctico (85\%, CAS 50-21-5), Zinc metálico usado para la policondensación con catalizador y Ácido Poliláctico (CAS 26100-51-6) que se aprovechó como estándar de comparación para los respectivos análisis de caracterización.

\section{B. Procedimiento}

El equipo que se empleó para la síntesis de PLA fue el Rotary Vacuum Evaporator - Boeco, modelo RVO 400 SD, en el cual se añade $40 \mathrm{~mL}$ de ácido láctico, trabajando a la agitación de 100 RPM y 100 mBar de presión, para posteriormente ser sometido a un proceso de calentamiento.

En la etapa de calentamiento, se incrementa la temperatura $15{ }^{\circ} \mathrm{C}$ en periodos de tiempo de 5 minutos, pudiendo observar que a la temperatura de $90{ }^{\circ} \mathrm{C}$ ocurre el proceso de destilación, dicho periodo de calentamiento permanece con las condiciones de presión y agitación ya mencionadas con anterioridad durante el periodo de polimerización.

Para la polimerización con catalizador, se alcanzó las temperaturas de 150,165 y $180{ }^{\circ} \mathrm{C}$, en tiempos de polimerización de 40,25 y 10 horas, respectivamente. Estas temperaturas se fijaron con la ayuda del termograma realizado al ácido láctico, el cual se detalla más adelante en la Fig. 1, mientras que los tiempos establecidos se determinaron experimentalmente.

Para la polimerización libre de catalizador, se alcanzó las temperaturas de 150,165 y $180{ }^{\circ} \mathrm{C}$, en tiempos de 50,48 y 30 horas, respectivamente.

La policondensación con catalizador, se efectúa con zinc metálico, en una concentración de $6 \%$ en peso, el cual se adiciona una vez que se alcanza las temperaturas propuestas con anterioridad.

El PLA que se sintetizó se pesó oportunamente para determinar el rendimiento del mismo, aplicando la ecuación 1 dada por [10], expresado como porcentaje en peso/peso:

$$
\text { Rendimiento de PLA(\%) }=\frac{\text { peso del material obtenido }}{\text { peso inicial de la muestra }} \cdot 100
$$

\section{Caracterización}

Tanto para la policondensación con catalizador como para la policondensación sin catalizador, con el objeto de presentar resultados claros y confiables, es necesario emplear técnicas de análisis para identificar los polímeros formados científicamente, razón por la cual se realizan los siguientes análisis:

\section{Espectroscopia FT-IR ATR}

Todos los espectros que se realizaron fueron analizados dentro de la región de 4000 a $600 \mathrm{~cm}^{-1}$ mediante espectroscopia infrarroja por la transformada de Fourier, el cual cuenta de un dispositivo ATR con cristal de diamante.

Por medio de este análisis se procede a la comparación de todas las muestras tomadas, para la posterior selección del polímero que presente mayor similitud respecto a los números de onda de los picos representativos del PLA de referencia, dicho análisis se lo emplea tanto para la policondensación con catalizador como sin catalizador.

\section{E. Termogravimetría (TGA)}

Se empleó el equipo Pyris 1 TGA para la realización de los análisis térmicos, colocando muestras de 3 y $4 \mathrm{mg}$ aproximadamente, dicho proceso fue llevado a cabo por calentamiento dinámico, el cual se analizó en un rango de temperatura de 0 a $500{ }^{\circ} \mathrm{C}$, a la velocidad de $10^{\circ} \mathrm{C} / \mathrm{min}$, bajo atmósfera inerte de nitrógeno.

Como resultado se tiene los termogramas, en donde se observa la gráfica porcentual de masa respecto la temperatura de calentamiento, detallándose las zonas de degradación de los polímeros formados para compararlos con la referencia de PLA.

\section{F. Calorímetro Diferencial de Barrido}

Los termogramas DSC se lo realizaron con ayuda del calorímetro diferencial de barrido DSC 1 STAR ${ }^{\circledR}$ System, siguiendo el procedimiento que recomienda el fabricante, en donde se utilizó como referencia una cápsula de aluminio $(40 \mu \mathrm{l})$ vacía sellada, el calentamiento de la muestra fue realizado a una velocidad de $10^{\circ} \mathrm{C} / \mathrm{min}$, en el rango de temperatura de $0{ }^{\circ} \mathrm{C}$ a $900{ }^{\circ} \mathrm{C}$, empleando un flujo de nitrógeno de $90 \mathrm{ml} / \mathrm{min}$. Mediante estos análisis es posible determinar el punto de fusión de las muestras obtenidas para ambos métodos, para posteriormente comparar dichas muestras con la referencia de PLA.

\section{ANÁLISIS DE RESULTADOS}

Para establecer los rangos de temperatura a los cuales se va a desarrollar los experimentos, es necesario en primera instancia realizar un termograma del ácido láctico que se emplea para la formación de PLA. Dicho termograma se presenta en la Fig. 1, en donde se aprecia que existe una pérdida de peso de ácido láctico entre las temperaturas de $149.28-220.45^{\circ} \mathrm{C}$, además, se identifica la degradación total del mismo por encima de $250{ }^{\circ} \mathrm{C}$.

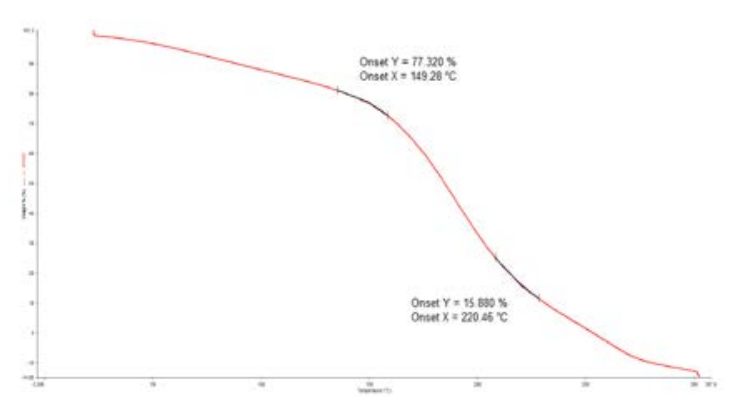

Fig. 1. Termograma del ácido láctico.

\section{A. Análisis espectroscópico FT-IR ATR}

La comparación de los análisis FTIR de los polímeros sintetizados en el laboratorio con el PLA de referencia, señalan que:

Mediante el método de policondensación con catalizador de zinc se obtiene PLA a la temperatura de $180^{\circ} \mathrm{C}$ a las 8 horas de polimerización,

Para el método de policondensación sin catalizador se 
obtiene el polímero biodegradable a la temperatura de 180 ${ }^{\circ} \mathrm{C}$ a las 20 horas de polimerización.

Los picos característicos de los grupos funcionales $\mathrm{O}-\mathrm{H}$, $\mathrm{C}-\mathrm{H}, \mathrm{C}=\mathrm{O}, \mathrm{CH}_{3}, \mathrm{C}-\mathrm{C}, \mathrm{C}-\mathrm{O}$ de la estructura del PLA se ubican en las longitudes de onda de 3506,65 - 2997,97 1750,33 - 1452,45 - 1182,90 - 1085,04 cm-1 respectivamente. Con la ayuda de los espectros FTIR de los polímeros obtenidos y la referencia de PLA, se identifica el polímero que presenta mayor coincidencia respecto a la referencia, es cual fue el obtenido mediante policondensación sin catalizador. En la Fig. 2 se evidencia el cambio que sufre la molécula de ácido láctico para dar la formación de ácido poliláctico.

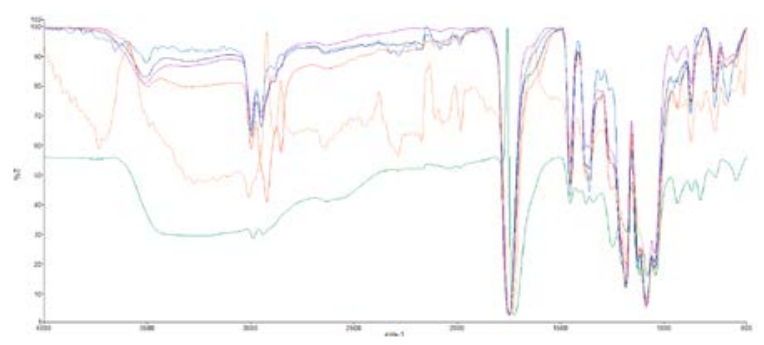

Fig. 2. Espectros FTIR.

\section{B. Análisis Termogravimétrico (TGA)}

Al analizar el PLA de referencia junto a los PLA sintetizados se identifican tres zonas que se muestran en la Fig. 3:

En la primera zona: existe pérdida de peso del polímero lo cual se da como resultado de las pérdidas de humedad, la volatilidad o por la presencia de compuestos que presentan pesos moleculares promedio.

En la segunda zona se aprecia la degradación térmica que ocurre en el polímero.

La descomposición de PLA ocurre en la tercera zona, en el rango de temperaturas entre 300 a $400{ }^{\circ} \mathrm{C}$, lo cual corrobora con los picos salientes reportados por el DSC.

Se distingue además que, el PLA obtenido por policondensación sin catalizador presenta una curva más cercana a la curva de referencia, lo cual indica que mencionado polímero es más estable térmicamente.

Por lo cual el PLA formado sin la adición de catalizador, al igual que en el análisis FTIR, presenta mayor similitud respecto al biopolímero que se elaboró con el uso de catalizador de zinc metálico.

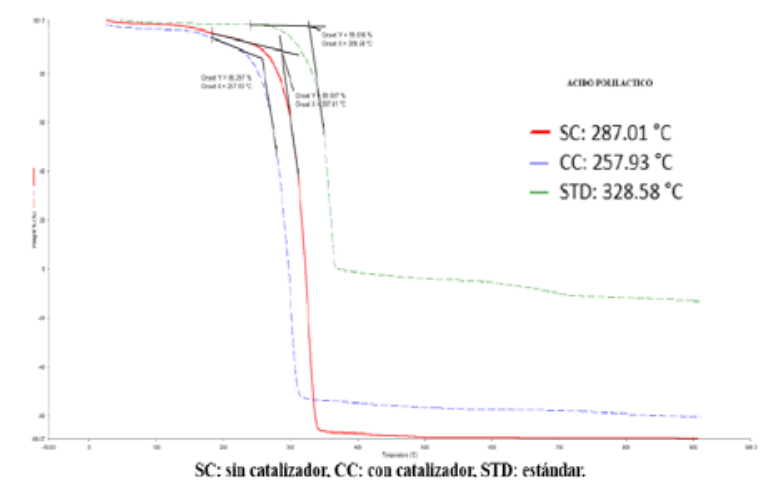

Fig. 3. Diagrama TGA.

\section{Calorimetría Diferencial de Barrido (DSC)}

Los picos que se observan en la Fig. 4 se encuentran en el rango de temperatura de 300 a $400{ }^{\circ} \mathrm{C}$, estos representan los puntos de fusión, los cuales son exotérmicos. Analizando cada una de las temperaturas de fusión de cada uno de los biopolímeros, se aprecia que los picos presentan variación en la amplitud de sus curvas, lo cual se interpreta como la diferencia de pesos moleculares de cada una de las muestras. Los termogramas DSC además reportan que a partir de la temperatura de $300{ }^{\circ} \mathrm{C}$ comienza la descomposición del PLA, lo cual también coincide con los termogramas realizados en el TGA.

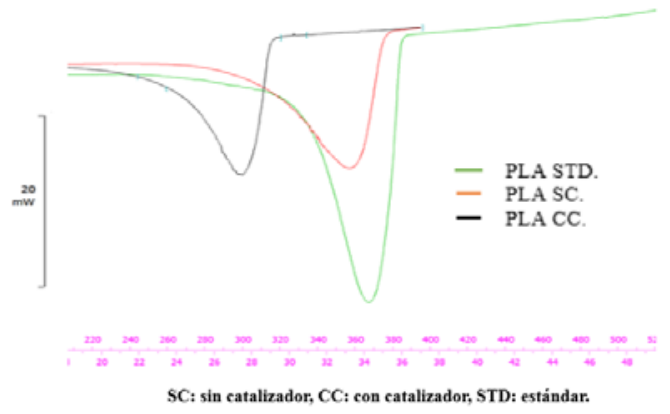

Fig. 4. Diagrama DSC.

Al comparar ambos polímeros sintetizados con la referencia de PLA, se indicar que, el polímero que presenta mayor coincidencia con el estándar es el sintetizado mediante el método de policondensación sin catalizador, lo cual corrobora con los análisis FTIR y TGA.

\section{Rendimiento.}

De los experimentos realizados se calcula el rendimiento obtenido en la síntesis de PLA con catalizador, el cual es de $67,53 \%$, mientras que el rendimiento para el método libre de catalizador es $68,83 \%$.

\section{CONCLUSIONES}

Debido a que no se requieren condiciones críticas de presión y temperatura, la producción industrial de PLA es factible utilizando el método de policondensación con catalizador y policondensación sin catalizador. Mediante el método de policondensación sin catalizador se logra obtener un PLA con mayores semejanzas al PLA de referencia, lo que se evidenció mediante los análisis FTIR, DSC y TGA.

\section{REFERENCIAS}

[1] Sánchez, L. W. L. (2015). Análisis de las políticas públicas sobre el cambio climático en el Ecuador.

[2] Orea, D. G., \& Villarino, M. T. G. (2013). Evaluación de impacto ambiental: Mundi-Prensa Libros.

[3] Diab, T., Biliaderis, C. G., Gerasopoulos, D., \& Sfakiotakis, E. (2001). Physicochemical properties and application of pullulan edible films and coatings in fruit preservation. Journal of the Science of Food and Agriculture, 81(10), 988-1000.

[4] Veloz, J., De La Torre, R., Ruiz Mendieta, J., \& Rivera Procel, S. (2009). Proyecto para reciclar el almidón de la yuca para la fabricación de fundas plásticas orgánicas.

[5] Zapata, D., Pujol, R., \& Coda, F. (2012). Polímeros biodegradables: una alternativa de futuro a la sostenibilidad de medio ambiente. Técnica Industrial, 297, 76-80.

[6] de la Torre-Cantero, J., Saorín, J. L., Meier, C., Melián-Díaz, D., \& Drago-Díaz Alemán, M. (2015). Creación de réplicas de patrimonio escultórico mediante reconstrucción 3D e impresoras 3D de bajo coste para uso en entornos educativos. Arte, individuo y Sociedad, 27(3). 
[7] Del Angel Lopez, d. (2009). Estudio y sintesis en la produccion de ácido polilactico.

[8] Serna, L., \& Albán, F. (2011). Ácido poliláctico (PLA): Propiedades y aplicaciones. Ingeniería y competitividad, 5(1), 16-26.

[9] Guanoluisa, C., \& Ismael, R. (2015). Obtención de Acido Poli Láctico a partir de lactosuero para la fabricación de empaque primarios. Escuela Superior Politécnica de Chimborazo.

[10] Arce, m. Á. V. Rendimiento y calidad de alginato del alga café eualaria fistulosa (postel y ruprecht) mj wynne de las islas aleutianas, Alaska. 